\title{
Report
}

\section{Recurrent Granulomatous Dermatitis with Eosinophilia}

\section{Wells' Syndrome}

\author{
Andrew J. MitChell, M.D., Thomas F. Anderson, M.D., \\ John T. Headington, M.D., and James E. Rasmussen, M.D.
}

From the Department of Dermatology, University of Michigan Medical Center, Ann Arbor, Michigan

\begin{abstract}
A 27-year-old woman developed a chronic, recurrent eruption of the face and upper extremities with the clinical and histopathologic features of recurrent granulomatous dermatitis with eosinophilia (Wells' syndrome). As described in 15 previously reported cases, this disorder is characterized by two clinical phases (eosinophilic cellulitis and granulomatous plaque phase) and three histopathologic stages. The latter are particularly remarkable for a diffuse dermal and subcutaneous eosinophilia in acute lesions and scattered flame figures in chronic lesions. Distinctive findings in this case were the predominance of facial involvement and the symptomatic response to topical corticosteroids. Although etiology and pathogenesis are unknown, we feel that Wells' syndrome is a unique yet rarely recognized clinicopathologic entity.
\end{abstract}

Recurrent granulomatous dermatitis with eosinophilia was first described by Wells. ${ }^{1}$ He reported four patients with an "obscure, recurrent dermatosis which combines a striking clinical picture with a very remarkable histopathology." 1 These patients had an eruption consisting of two clinical phases. The first phase, designated an eosinophilic cellulitis, was described as a rapidly spreading, poorly demarcated area of erythema and edema that occasionally blistered. The inflammation could involve a small area on the trunk or an extremity or expand to involve an entire limb. In the second phase, occurring

Address for reprints: Andrew J. Mitchell, M.D., Department of Dermatology, Henry Ford Hospital, Detroit, MI 48202.
1-3 weeks following the onset of eosinophilic cellulitis, there was a gradual metamorphosis of the acute "cellulitis" into a plaque of indurated, slate-colored skin. Eventually these skin changes completely resolved without residual scarring.

In this report we describe an additional case of recurrent granulomatous dermatitis with eosinophilia. Our patient both reinforces and augments the spectrum of cutaneous changes that can occur in this disorder. We feel that the clinical and histopathological findings in Wells' syndrome are unique and yet rarely recognized in the American literature.

\section{Case Report}

A 27-year-old woman was referred to the University of Michigan Department of Dermatology with a one-month history of a pruritic, scaly, red swelling over the right side of her chin and jaw. She had also noted occasional transient "welts" on the face during the previous several months, each lasting less than 12 hours. The only medications had been topical clindamycin hydrochloride $1 \%$ in hydro-alcoholic solution and $5 \%$ benzoyl peroxide gel for mild acne vulgaris.

Past medical history and review of systems were unremarkable. In particular, there had been no constitutional symptoms.

At the time of her initial visit to this clinic, the patient appeared to be in good health. She had an $8-10 \mathrm{~cm}$ area of indurated swelling over the right side of the mandible (Fig. 1). The overlying skin appeared eczematous with erythema, scale, and crust. The results of the remainder of the physical examination were within normal limits. The initial differential diagnosis included allergic contact dermatitis, angioedema, and cellulitis. All topical agents were discontinued, and the patient's dermatitis resolved with a two-week course of oral erythromycin and prednisone $(40 \mathrm{mg} / \mathrm{po} / \mathrm{qd}$ for 7 days and $20 \mathrm{mg} / \mathrm{po} / \mathrm{qd}$ for 7 days).

The patient returned in one month with a recurrence of erythema and edema of the right cheek and jaw (10-12 cm area). The periphery of the lesion was s:ightly violaceous. An- 
FIG. 1. Eosinophilic cellulitisacute phase lesion with erythema, edema, and scaling over the mandible.

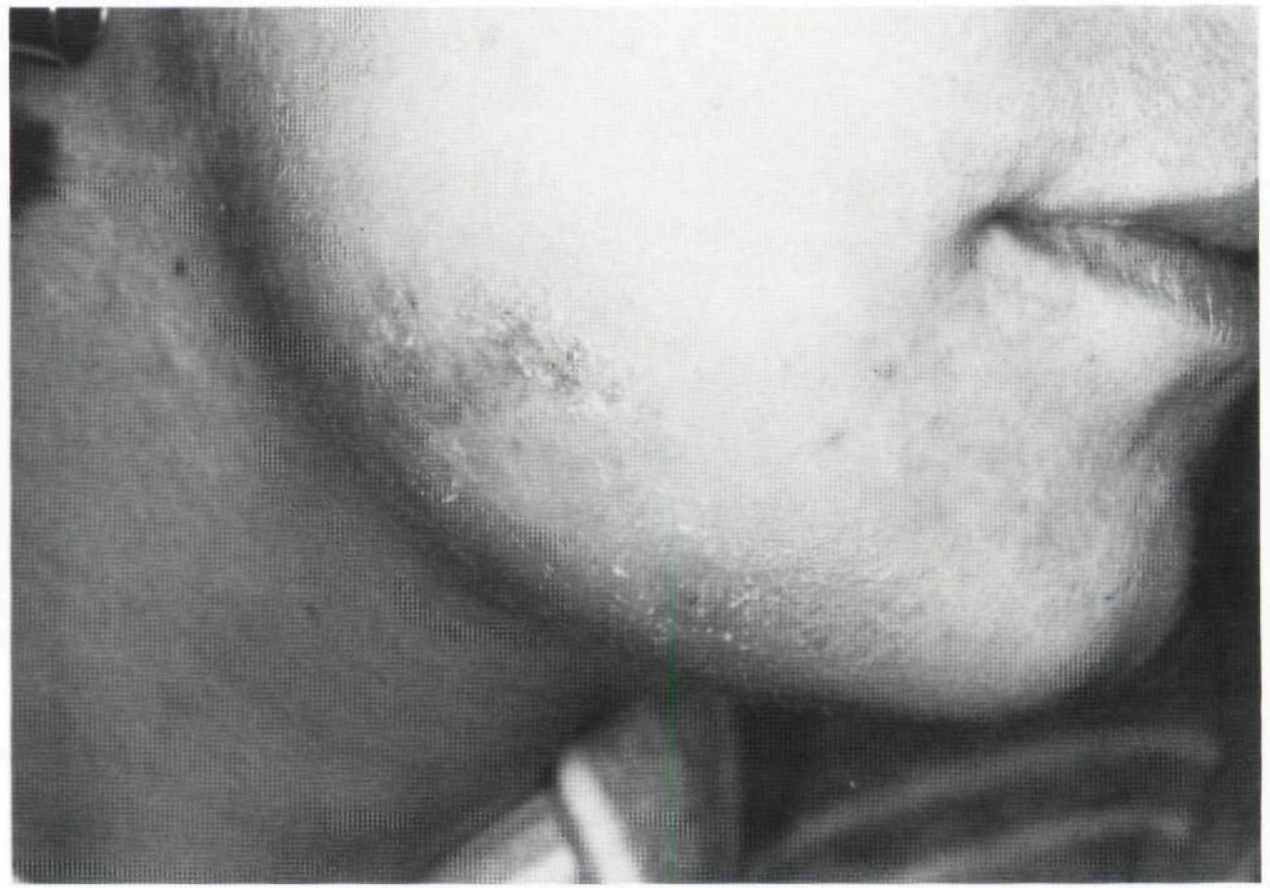

gioedema was suspected, so the patient was placed on a tartrazine/salicylate-free diet, asked to keep a dietary log, and treated with hydroxyzine and prednisone for two weeks. The acute swelling resolved within one week, but an area of slight induration with a grayish hue persisted. The latter changes disappeared in approximately three weeks.

During the following four months, the patient continued to have intermittent recurrences of right facial swelling, often with overlying eczematous changes. Similar, although smaller, lesions occurred on the arms and shoulders without detectable dietary or other precipitant. The eruptions continued to respond to short courses of oral corticosteroids. Residual morphea-like induration would persist for several weeks following each acute lesion. Our differential diagnosis expanded to include urticarial vasculitis and Wells' syndrome. During this period of observation, laboratory evaluation found the following studies to be normal: serum electrolytes, creatinine, blood urea nitrogen, calcium, phosphorus, serum glutamic oxaloacetic transaminase, lactic acid dehydrogenase, alkaline phosphatase, $\mathrm{C}_{3}$ and total hemolytic complement, blood glucose, white blood cell count and differential count, hemoglobin, platelet count, urinalysis, antinuclear antibody, sedimentation rate, and $x$-rays of chest and mandible. Two eosinophil counts $\left(229 / \mathrm{mm}^{3}\right.$ and $\left.160 / \mathrm{mm}^{3}\right)$ were within normal limits. These were obtained during flares of the disorder when the patient was not on corticosteroids. Granulocyte function studies (myeloperoxidase activity, nitroblue tetrazolium test, bactericidal activity against Staphylococcus aureus and E. coli, and chemotactic migration) were normal. Immunofluorescence of a biopsy specimen from a nodule (approximately one-week old) on the left shoulder was negative for $\lg G, \lg A$, $\lg \mathrm{M}, \mathrm{C}_{3}$, and fibrin.

Biopsies for routine histopathology were taken from an acute swelling over the right mandible and a three-week-old plaque on the left forearm.
The patient was lost to follow up because of a permanent move away from this area. In the six months that she was under our care, her cutaneous eruptions became less frequent and less severe. The occasional nodule that occurred on the shoulder or arm responded well to topical fluocinonide cream $(.05 \%)$, and systemic therapy was not necessary during this period.

\section{Histopathologic Findings}

Histologic studies included biopsy samples of both acute and subacute phase lesions.

Epidermal changes in the acute phase (Fig. 2) included slight orthokeratotic hyperkeratosis, slight unpatterned acanthosis, and slight diffuse spongiosis with occasional intraepidermal lymphocytes and eosinophils. A few lymphocytes and eosinophils were found in an edematous papillary dermis.

Reticular dermis and subcutis were most extensively involved by inflammation. Edema of the deep reticular dermis with striking separation of collagen was associated with a marked interstitial infiltrate composed of eosinophils and lymphocytes (Fig. 2). Hypertrophy and basophilia of dendritic dermal histiocytes contributed a striking additional cellular component. A marked angiocentric infiltrate of eosinophils and lymphocytes in the vascular adventitia added another dimension to the histologic pattern.

Dermal inflammation was carried well into the subcutis with extensive interstitial inflammation of fatty 


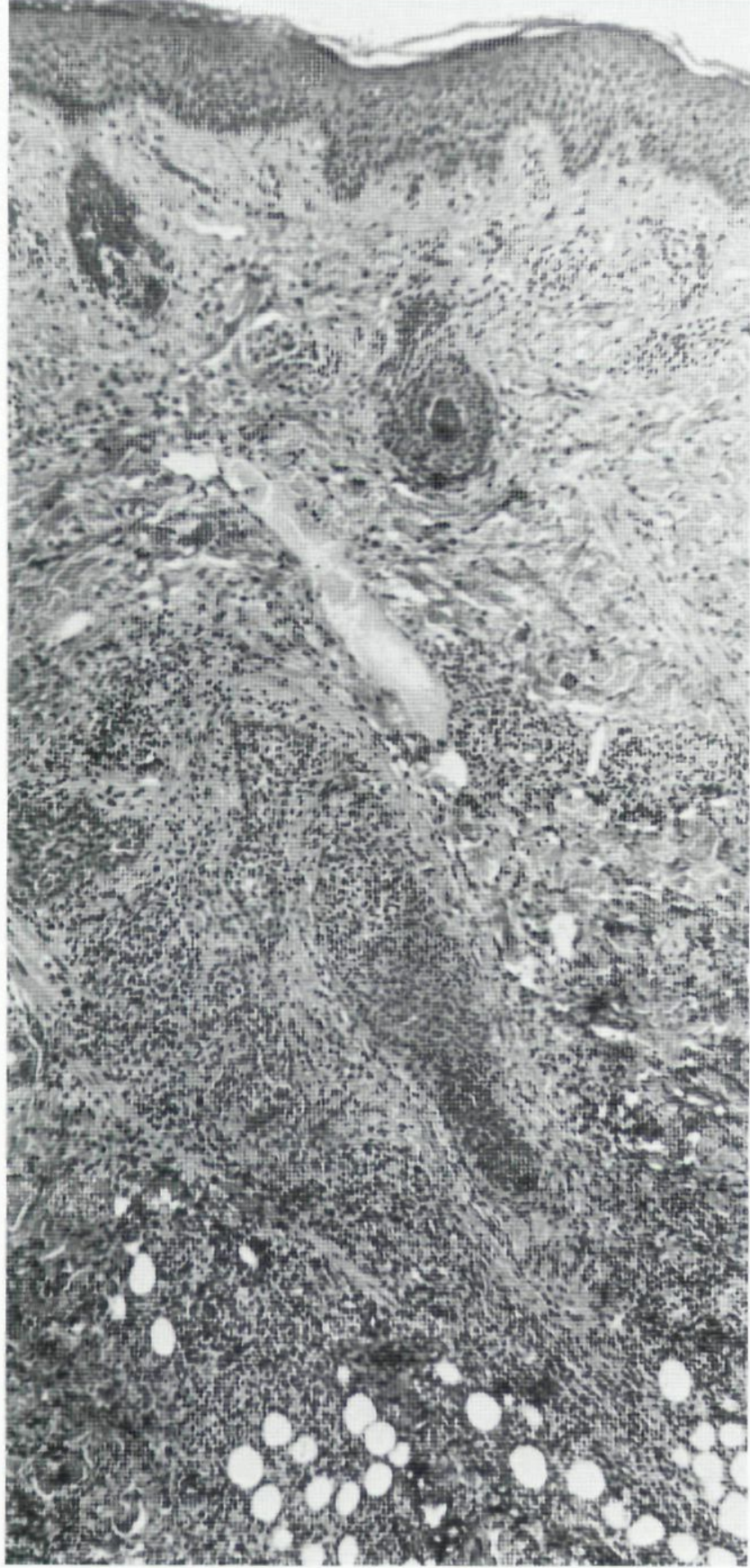

FiG. 2. Eosinophilic cellulitis-acute phase lesion. Note slight orthokeratotic hyperkeratosis and diffuse spongiosis. A marked interstitial and angiocentric infiltrate of eosinophils and lymphocytes is found in dermis with extension into subcutis. (H\&E, original magnification, $\times 60$ ).

lobules but without necrosis of adipocytes. The pattern of involvement of the subcutis was mixed septal and lobular.

Vellus hair follicles included in the biopsy were shifted into telogen with slight mucinous transformation of outer sheath epithelium, somewhat similar to that seen in early stage alopecia mucinosa.
There was no evidence for vasculitis, and karyorrhexis of polymorphonuclear leukocytes was not seen. Margination of eosinophils, migration of eosinophils through vessel walls, and endothelial cell hypertrophy was largely confined to small vessels, probably venules. Flame figures were not found in this stage.

In the subacute phase epidermal changes were somewhat more marked with the appearance of parakeratosis. Small papillary vessels were congested. Angiocentric inflammatory infiltration persisted, but interstitial inflammation was reduced compared with the acute phase. Prominent flame figures (Fig. 3) were composed of aggregates of eosinophils, extracellular eosinophil granules, and eosinophilic reticular collagen.

\section{Discussion}

In this paper we describe a patient with the clinical and histopathologic findings of recurrent granulomatous dermatitis with eosinophilia. As first characterized by Wells, ${ }^{1}$ and in subsequent reports by Wells and Smith, ${ }^{2}$ and Spigel and Winkelmann, ${ }^{3}$ this eruption was noted to have two phases. The first phase was designated an eosinophilic cellulitis and consisted of a rapidly spreading patch or plaque of erythema and edema with occasional vesiculation. As it spread, the erythema tended to fade from the center of the lesion. The inflammation typically involved a small area on the trunk or an extremity, but occasionally expanded to involve an entire limb. In one of Wells' original four cases, facial lesions were noted late in the course of the disorder. In this first stage, itching, burning, and/or pain were experienced at various times. The second phase appeared as the acute "cellulitis" faded, approximately $1-3$ weeks following disease onset. The affected area became indurated and slate-colored, frequently giving the impression of atrophy and occasionally the appearance of morphea. This "granulomatous" phase resolved completely in a period of approximately six weeks.

All four of Wells' original cases had recurrent nonspecific cutaneous eruptions, often urticarial, prior to the onset of eosinophilic cellulitis. ${ }^{1}$ In only one of the four patients was there the suggestion of a precipitating event (penicillin treatment for pharyngitis). In the two cases of Spigel and Winkelmann, ${ }^{3}$ there was again the implication of a possible relationship to drugs. The age of onset in these six cases ranged from 26 to 71 years. The female to male ratio was $2: 1$.

The disorder tended to be chronic but in nearly all cases showed a tendency toward gradual spontaneous resolution over several years. Successful suppression of the eruption with Prednisone was described in four instances. Laboratory evaluation revealed a peripheral eosinophilia in all six cases. However, Wells and Smith, ${ }^{2}$ in a brief report of seven additional cases of eosinophilic 


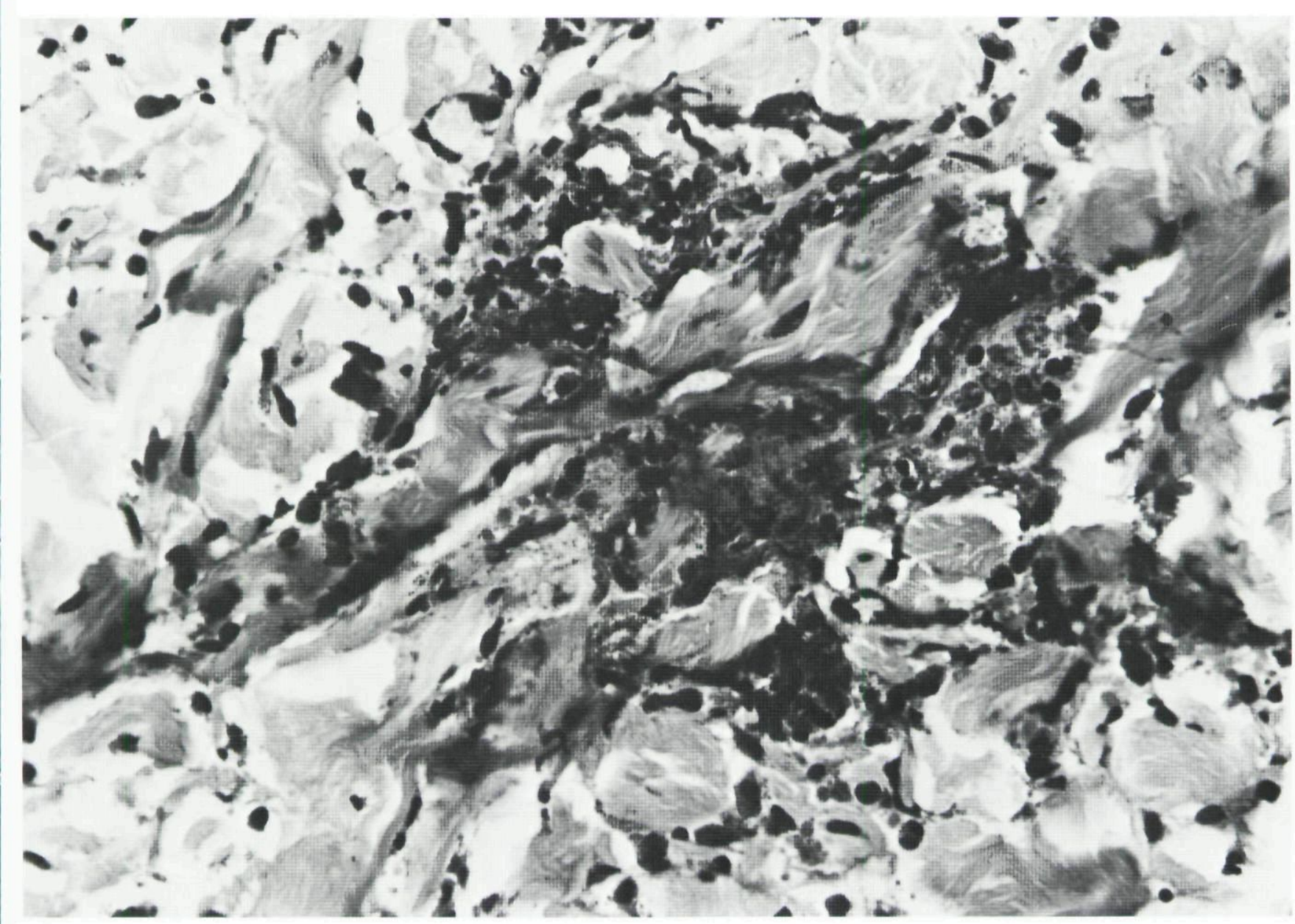

FIG. 3. Subacute lesion (3 weeks). Note flame figure, an aggregate of lymphocytes and histiocytes surrounding a core of eosinophils, extracellular eosinophil granules, and eosinophilic collagen. (H\&E, original magnification, $\times 120$ ).

cellulitis noted that only half of these patients exhibited peripheral eosinophilia. In general, constitutional symptoms associated with Wells' syndrome were not observed, although an atopic history was a frequent finding. ${ }^{4}$ A patient recently described by Marks ${ }^{5}$ was notable for her history of asthma as well as her apparent response to treatment with Dapsone. Eosinophilic cellulitis also has been recently reported in a child, and in this case, fever and arthralgias were associated findings. ${ }^{6}$

Histopathologic changes were divided into three stages. Corresponding to the first clinical phase, the acute histologic stage was termed eosinophilic cellulitis. It was characterized by dermal edema and infiltration with leukocytes, predominately eosinophils. These cells were seen both in a perivascular position as well as more diffusely throughout the dermis. There were no destructive changes in vessel walls. The occasional blister was noted to be subepidermal.

The second or subacute histopathologic stage corresponded to the later clinical phase when skin had become indurated and less inflamed. The characteristic finding was that of a diffuse dermal granulomatous infiltrate composed of histiocytes and eosinophils. An additional striking finding in this stage was the "flame figure," an aggregate of pallisading histiocytes and giant cells surrounding a core of collagen coated with eosinophilic debris. These figures were found scattered in the mid-and deep dermis. The third pathologic phase (stage of resolution) occurred later in the clinical course, when the skin had attained an atrophic and grayish appearance. At this point there were fewer eosinophils, but histiocytes and giant cells persisted diffusely between collagen bundles. Flame figures remained and occasional "necrobiotic" foci, similar to those in granuloma annulare, were observed.

Several questions are germane to the remarkable histology of eosinophilic cellulitis. An important consideration is whether the histologic changes represent an unusual but nonspecific reaction phenomenon- a final common pathway event, or whether there is a uniqueness that permits accurate and reproducible recognition as a clinicopathologic entity. The only morphologic 
point that is unequivocally clear is that the flame figures distinguishing the subacute lesion are distinctly nonspecific and may be found in several quite different disorders (bullous pemphigoid, dermatophytosis).

The epidermal changes, increased dermal hyaluronic acid, increased telogen phase hair follicles, and partial mucinous transformation of the tricholemma all appear to be secondary events.

Our patient displayed clinical and histopathologic findings very similar to those described by Wells, ${ }^{1}$ Wells and Smith, ${ }^{2}$ and Spigel and Winkelmann. ${ }^{3}$ She had no suggestion of a drug reaction or other specific precipitating event. Her long history of a preceding recurrent urticarial eruption resembled the frequent finding of antecedent nonspecific rashes in many of Wells' patients. Also similar to previously reported cases was the initial differential diagnosis, which included angioedema, contact dermatitis, and cellulitis. Finally, her response to oral corticosteroids and her gradual spontaneous improvement over the course of a year were both compatible with the observations of others. Findings that were not typical of previous cases were the severe acute facial involvement as a presenting sign and the apparent symptomatic response to topical corticosteroids. The absence of peripheral eosinophilia cannot be considered atypical, as half the cases reported by Wells and Smith ${ }^{2}$ were lacking this finding.

The etiology of recurrent granulomatous dermatitis with eosinophilia is unknown. A role for allergic hypersensitivity in the pathogenesis is suggested by the common association with atopic disorders, the suggestion of drug-related precipitation in some cases, and the frequent presence of peripheral eosinophilia. The extraordinary localized tissue eosinophilia is of primary importance because it is doubtful that the pathogenesis of the lesion will be explained without an explanation for the marked eosinophil chemotaxis. As an immunologic event, there is no established histologic paradigm, but an immunologic reaction is not improbable. Is it, as Wells suggests, eosinophil cytotoxicity mediated by $\lg \mathrm{G}$, or is there a subset of effector lymphocytes or mast cells that produces an eosinophil promoting factor?

Further investigation of the relative contribution of type I, type III, and/or type IV hypersensitivity in Wells' syndrome would be of value. In particular, measurement of soluble mediators, such as the eosinophil chemotactic factor of anaphylaxis may prove useful. The latter has been measured by Soter et $\mathrm{al}^{7}$ in their demonstration of mast cell mediator release in cold urticaria.

Another area of potential pathogenetic significance concerns the possible role of immune complexes. Direct immunofluorescence of involved skin revealed a positive reaction for $\mathrm{C}_{3}$ in walls of dermal vessels in one of Wells' cases $^{4}$ and showed deposition of $\lg \mathrm{M}, \lg \mathrm{A}, \lg \mathrm{D}$, and $\mathrm{C}_{3}$ around dermal vessels in the patient described by Marks. ${ }^{5}$ In our case, direct immunofluorescence was negative. However, the lesions sampled were more than one-day old, and the presence of immunoglobulins may therefore have been missed. Attempts at induction of an incipient lesion with intradermal histamine injection might be more likely to reveal early immunoglobulin and complement deposition in vessel walls, as has been demonstrated by Braverman and Yen ${ }^{8}$ in the study of leukocytoclastic vasculitis. Clarification of pathogenesis will have to await description and analysis of additional cases of granulomatous dermatitis with eosinophilia.

\section{Drug Names}

clindamycin: Cleocin

hydroxyzine: Atarax, Vistaril

\section{Acknowledgment}

James Stroud, M.D. referred this patient.

\section{References}

1. Wells GC: Recurrent granulomatous dermatitis with eosinophilia. Trans St Johns Hosp Dermatol Soc 57:46, 1971

2. Wells GC, Smith N: Eosinophilic cellulitis-Persistent cutaneous swelling with phagocytosis of eosinophilic debris (abstracted). Br J Dermatol 99 (suppl. 16):11, 1978

3. Spigel GT, Winkelmann RK: Wells' syndrome. Recurrent granulomatous dermatitis with eosinophilia. Arch Dermatol $115: 611,1979$

4. Wells GC: Eosinophilic cellulitis. In: Vasculitis (Series Major Problems in Dermatology, vol 10). Edited by Wolff K, Winkelmann RK. Philadelphia, WB Saunders, pp 317-321

5. Marks R: Eosinophilic cellulitis-A response to treatment with dapsone: Case report. Australas J Dermatol 21:10, 1980

6. Nielsen T, Schmidt H, Sogaard H: Eosinophilic cellulitis (Wells syndrome) in a child. Arch Dermatol 117:427, 1981

7. Soter NA, Wasserman SI, Austin KF: Cold urticaria. Release into the circulation of histamine and eosinophil chemotactic factor of anaphylaxis during cold challenge. N Engl J Med 294:687, 1976

8. Braverman IM, Yen A: Demonstration of immune complexes in spontaneous and histamine-induced lesions and in normal skin of patients with leukocytoclastic angiitis. I Invest Dermatol $64: 105,1975$ 
This document is a scanned copy of a printed document. No warranty is given about the accuracy of the copy. Users should refer to the original published version of the material. 